\title{
Isolation of persistent air leaks and placement of intrabronchial valves
}

Amit K. Mahajan, MD, Diana C. Doeing, MD, and Douglas K. Hogarth, MD

\begin{abstract}
Objectives: Alveolar-pleural fistulas causing persistent air leaks are conditions associated with prolonged hospital courses, high morbidity, and possibly increased mortality. Intrabronchial valves serve as a noninvasive therapeutic option for the closure of alveolar-pleural fistulas.
\end{abstract}

\begin{abstract}
Methods: The present review describes a brief history of, and indications for, the placement of intrabronchial valves in patients with persistent air leaks. The essential steps necessary for placement are air leak isolation, airway sizing, and valve deployment. Additionally, the indications and methods for intrabronchial valve removal, along with the potential complications from intrabronchial valve placement, are described.
\end{abstract}

Conclusions: The increased use of intrabronchial valves in the treatment of persistent air leaks requires bronchoscopists and clinicians to understand the procedural steps and techniques necessary for intrabronchial valve placement. (J Thorac Cardiovasc Surg 2013;145:626-30)

Intrabronchial valves (IBVs) are small umbrella-shaped devices designed to limit airflow to distal portions of the lung. The IBVs are placed into the desired airways using a flexible bronchoscope and are removable. Although a number of recent studies have described the utility of IBVs for the treatment of persistent air leaks (PALs) after alveolar-pleural fistulas (APFs) due to pneumothoraces, a clear explanation of placement techniques have rarely been reported in detail. The present report describes the step-by-step process to isolate the air leaks and then properly place the IBVs.

Bronchial valves were initially developed as an investigational approach for the bronchoscopic treatment of emphysema by lung volume reduction. Designed to isolate the airway segments, the valves induce atelectasis in the emphysematous lung segments. These studies involved 2 types of airway valves: the IBV system (Spiration, Redmond, Wash) and the Zephyr (Emphasys, Redwood City, Calif). ${ }^{1-3}$ Although study of the Emphasys valves in the Endobronchial Valve for Emphysema Palliation Trial (VENT) showed improvements in lung function, exercise tolerance, and symptoms, an increased incidence was found in the exacerbation frequency of chronic obstructive pulmonary disease, an increased incidence of pneumonia, and an increase incidence of hemoptysis after implantation. ${ }^{4}$ Currently, Zephyr valves are not approved for use in the United States. Spiration IBVs continue to be studied for use in severe emphysema and are considered investigational for the management of emphysema.

From the University of Chicago Medical Center, Chicago, Ill.

Disclosures: Authors have nothing to disclose with regard to commercial support.

Received for publication Oct 5, 2012; revisions received Nov 20, 2012; accepted for publication Dec 3, 2012; available ahead of print Jan 10, 2013.

Address for reprints: Amit K. Mahajan, MD, University of Chicago Medical Center, 5841 S Maryland Ave, MC 6076, Chicago, IL 60637 (E-mail: amit.mahajan@ uchospitals.edu)

0022-5223/\$36.00

Copyright $(2) 2013$ by The American Association for Thoracic Surgery

http://dx.doi.org/10.1016/j.jtcvs.2012.12.003
During the clinical trials of both the Zephyr and the Spiration valves, several valves were placed in compassionate use scenarios to control PALs. Clinical series by Gillespie and colleagues ${ }^{5}$ and Travaline and colleagues ${ }^{6}$ have demonstrated the safe and efficacious use of these technologies to aid in the management of PALs resulting from APFs. Although they have not been studied in randomized controlled studies, the IBV system has been approved through the Humanitarian Device Exemption for PALs after segmentectomy, lobectomy, and lung volume reduction surgery. Additionally, a number of physicians have used IBVs in the off-label treatment of APFs to correct PALs and even aid in weaning from significant oxygen support and mechanical ventilation in intensive care unit patients. ${ }^{7,8}$

Bronchopleural fistulas are pathologic communications between the bronchial tree and the pleural space, and APFs are pathologic communications between the alveoli and the pleural space. These communications can arise from a number of conditions, including, but not limited to, pneumothoraces, pulmonary infections, trauma, malignancies, and complications after thoracic surgery. Both of these processes can result in the creation of PALs that can result in high morbidity, increased mortality, and prolonged hospitalization. ${ }^{9-11}$ A number of therapeutic options, both invasive and noninvasive, are available for the treatment of PALs secondary to bronchopleural fistulas. Noninvasive approaches to therapy include prolonged chest tube drainage with tailored ventilator strategies aimed at establishing acceptable ventilation and oxygenation while reducing flow through the fistula. ${ }^{12,13}$ Invasive therapies include pleural abrasion, application of fibrin sealant, bronchial stump stapling, muscle flap construction, omental flap coverage, and surgical lobectomy. The use of devices previously used in vascular surgery, such as Amplatzer vascular plugs, are even being explored in the treatment of PALs. ${ }^{14} \mathrm{~A}$ 2001 American College of Chest Physicians consensus 

Abbreviations and Acronyms
$\mathrm{APF}=$ alveolar-pleural fistula
$\mathrm{EBV}=$ endobronchial valve
IBV = intrabronchial valve
PAL = persistent air leak
VENT $=$ Endobronchial Valve for Emphysema Palliation Trial

\section{OVERVIEW OF IBV PLACEMENT}

The placement process of IBVs is a collection of steps aimed at identifying and occluding the lung segments contributing to the PALs. These steps include air leak isolation, airway sizing, and valve deployment.

\section{Air Leak Isolation}

Identifying the offending segment containing the PAL is a critical step in the placement of IBVs. Although it might seem intuitive to the treating physician that one should "know" where an air leak is originating, frequently more than 1 airway is involved in the PAL. Because the IBV isolates that region of the lung, it is imperative to know which segments require valve placement to avoid occlusion of viable lung not involved in the PAL.

Several methods for air leak isolation have been described but the most reliable continues to be sequential balloon occlusion of segmental airways, moving proximally to distally. This is accomplished by using a simple balloon catheter. We begin by first occluding each mainstem bronchus to demonstrate whether we can truly "stop" the air leak. We then proceed distally and occlude the whole upper lobe. If no change occurs in the degree of the air leak, we isolate the lower lobe (and if on the right, the middle lobe at the same time). Once we have identified the target lobe, we test each individual segment. This systematic approach allows the detection of complex air leaks involving more than 1 segment and/or more than 1 lobe. Various other methods have been studied in an effort to isolate PALs, such as the use of nuclear medicine, selective bronchography, and exhaled volumes on the ventilator (D. FellerKopman, personal communication, May 2012). ${ }^{15,16}$

During air leak isolation, we recommend at least an $8.5 \mathrm{~F}$ or larger endotracheal tube to prevent "artificial" occlusion of the air leak by the bronchoscope. The current IBV system available in the United States requires a $2.8-\mathrm{mm}$ working channel (the European system requires a $2.0-\mathrm{mm}$ working channel). Visualization of the pleural drainage device should be arranged so the interventional team can search for reduction in the air leak during occlusion of the suspected airways. During each occlusion, it is recommended to wait several respiratory cycles (roughly 5 breaths) to determine the effect of airway occlusion on the air leak. After occlusion, residual air remains in the distal portion of the airway and in the pleural space. After 4 to 5 breath cycles, the air should be evacuated from the pleural space, allowing the operator to judge whether a reduction of airflow through the defect has truly occurred. A reduction in the size of the air leak should indicate that the operator is following the correct path to the involved segment, and an increase in the air leak should represent redistribution of more air from a normal segment through the defect.

The source of air leaks can be quite variable depending on the precipitating event that induced the PAL. The 

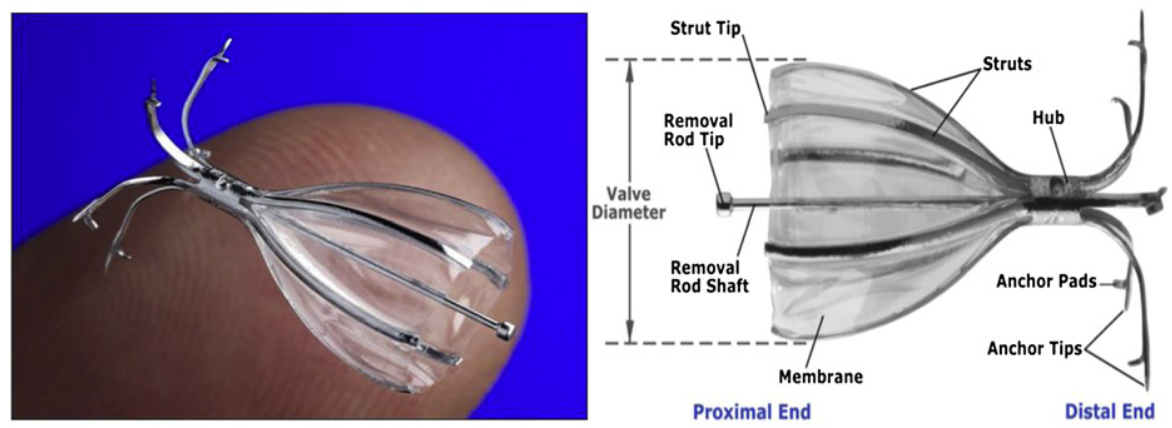

FIGURE 1. A, Close-up view of intrabronchial valve. B, Close-up view, labeled. Provided by Spiration Inc (Redmond, Wash).

presence of numerous defects, especially involving multiple segments or multiple lobes, often involves prolonged procedural times for isolation and might ultimately require deployment of several valves. In our experience, our patients have required 2 to 9 valves to control PALs.

\section{IBV Sizing}

Once the air leak has been isolated, the first step is to size the airway to determine the correct IBV to be placed. The IBV system comes with an airway sizing kit that calibrates a balloon catheter (Olympus B5-2C balloon catheters or Applied Medical balloon catheters, Python EC $11 \mathrm{~mm} / 5 \mathrm{~F}$ ) to measure the airway diameter and determine the valve size for deployment. The deflated balloon should be advanced to the opening of the airway and slowly inflated until the balloon has made gentle contact with the airway walls (Figure 2). Once the balloon has been inflated and made circumferential contact with all portions of the airway wall, an attempt should be made to advance and withdraw the balloon gently to ensure no migration occurs distally or proximally of the balloon within the airway. An appropriate inflation volume should provide the beginning of drag on the airway with movement of the balloon. The balloon should not be overinflated causing dilation or distension of the airway. The degree of balloon inflation should correspond to the valve size. Inaccurate sizing of the airway can result in a poor seal of the airway and allow the PAL to continue.

\section{IBV Placement}

Valve deployment is a multistep process using the IBV catheter. Once the single-use valve is loaded, the catheter tip should be inspected to guarantee the valve has been appropriately loaded and no valve anchors are protruding from the catheter. With the bronchoscope resting in a more proximal, larger airway, the catheter is advanced out of the bronchoscope so the compressed valve can been visualized. The stabilization rod is separated from the stabilization wire by roughly $1 \mathrm{~mm}$, requiring the operator to "close the gap" to improve the deployment accuracy and prevent kinking of the catheter (Figure 3). With the bronchoscope in the neutral position, the safety clip should be removed from the most proximal portion of the catheter, and the catheter handle should be gently pressed to eliminate the space between the removal rod and stabilization wire. The catheter and valve should then be retracted into the bronchoscope. At this point, the bronchoscope can be directed to the target airway. Once the target airway has been visualized, the catheter should be advanced distally to the target location, with constant attention paid to the black valve line signifying the deployment site. The catheter is then pulled proximally to align the valve line with the desired valve placement location. At the end of maximum inhalation, the retractor handle should be squeezed gently with persistent pressure to deploy the IBV into place. Valve deployment should be performed in 1 continuous motion within 1 to 2 seconds. The deployed valve should be observed after placement into
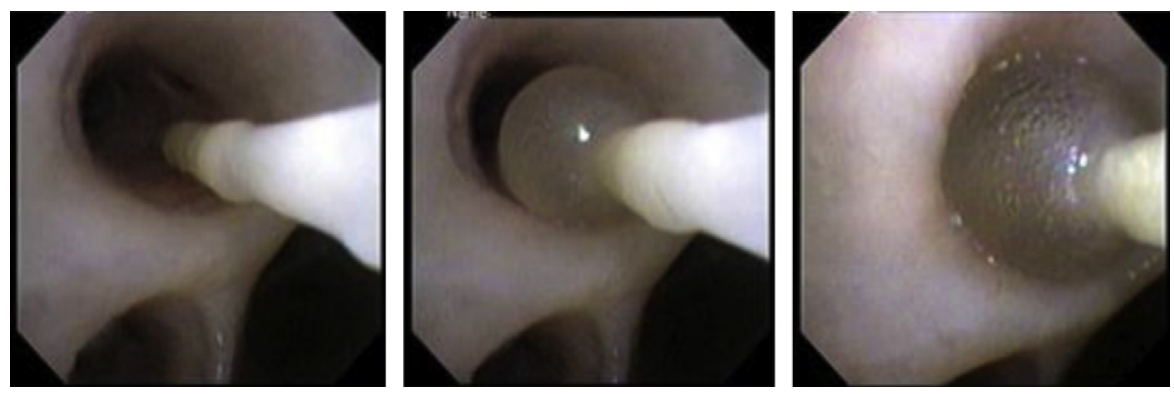

FIGURE 2. Diagram showing balloon occlusion. Provided by Spiration Inc (Redmond, Wash). 


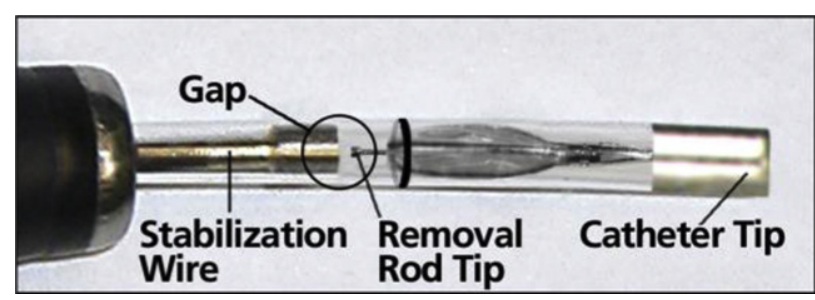

FIGURE 3. Diagram showing closing the gap. Provided by Spiration Inc (Redmond, Wash).

the airway for 25 to 30 seconds to ensure proper positioning (Figure 4). After valve placement, the air leak chamber of the chest tube collecting system should be observed for 4 to 5 ventilatory cycles to assess any changes in the degree of the air leak. During this period, occlusion of the culprit airway might divert airflow to other areas of the lung, unmasking additional air leaks that might not have been appreciated during the air leak identification process. These steps should be repeated as necessary for each segment needing valve placement.

\section{IBV Removal}

The criteria for IBV removal vary and depend on clinical judgment. Resolution of air leaks with adequate time for tissue healing is a well-accepted indication for IBV removal. Physicians should re-evaluate patients approximately 6 weeks after valve placement to determine whether IBV removal is a feasible option. Additionally, if surgical intervention is planned because of an inability to correct the PAL using IBVs, all valves should be removed bronchoscopically before surgery. Removal of the IBVs should be performed using either cupped or rat-toothed forceps. The use of an endotracheal tube is recommended to facilitate ventilation and anesthesia and, most importantly, to protect the vocal cords and other upper airway structures from damage as the IBVs are withdrawn from the patient. IBV removal does not require a $2.8-\mathrm{mm}$ working channel, because the valves are not drawn into the bronchoscope channel. The removal of the IBVs requires the bronchoscopist to grasp the removal rod shaft and gently pull proximally until the IBV has been dislodged from the airway wall (Figure 5). Before removal of the IBV into the endotracheal tube, the IBV should be pulled as close as possible to the end of the bronchoscope. The bronchoscope and forceps should be pulled out simultaneously through the endotracheal tube. In the setting of a normal coagulation panel, only minimal bleeding should occur during the removal process.

\section{Complications of IBV Placement}

The list of complications from IBV placement is a composite of the complications observed during clinical trials focusing on lung volume reduction for emphysema, because no specific trials have been conducted to observe placement of IBVs for PALs. The possible complications are listed in the "Instruction for Use" packet included with the IBV system (Table 1). The VENT trial succeeded in confirming a favorable safety profile for the use of endobronchial valves, showing that by 6 months, the rate of the composite of 6 major complications was $6.1 \%$ in the endobronchial valve (EBV) group and $1.2 \%$ in the control group $(P=.08)$, a level that met the prespecified safety criteria. ${ }^{4}$ Additionally, follow-up through 12 months continued to show a complication profile that was not significant between the EBV and control groups. The most common adverse event observed in the VENT trial were pneumonia in the segment distal to valve placement. All cases of pneumonia resolved with the use of antibiotics, with 6 patients requiring hospitalization, none of whom required mechanical ventilation. Additionally, the presence of hemoptysis (defined as bleeding requiring bronchoscopic inspection) during both early (within 90 days of the procedure) and late (3-12 months after the procedure) follow-up was significantly increased in the EBV

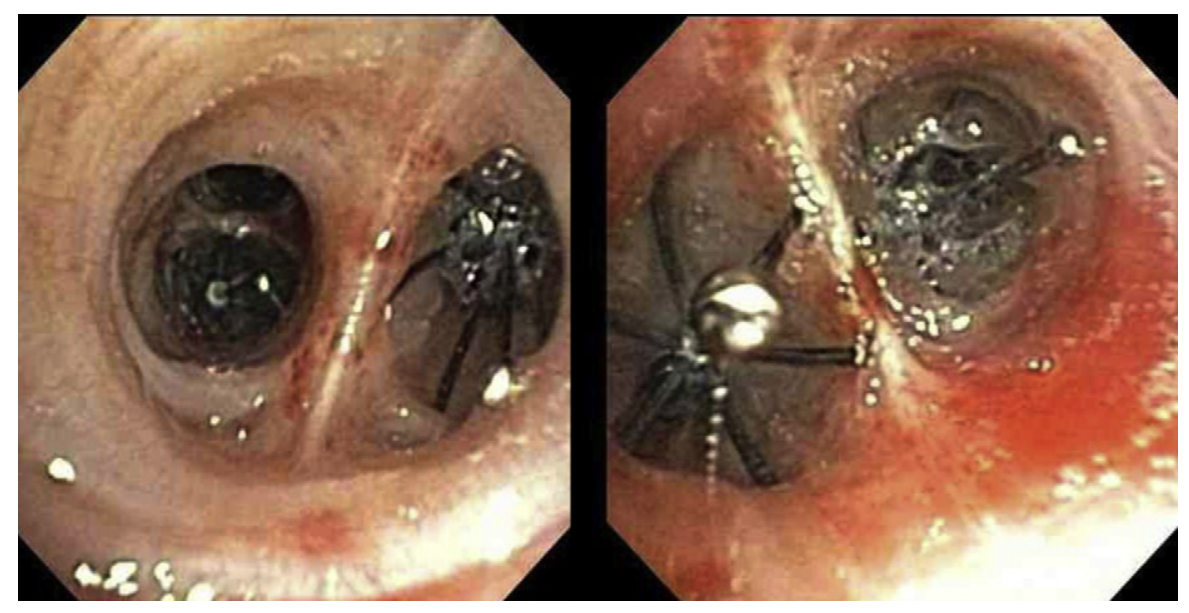

FIGURE 4. A and B, Endoscopic views showing valve in airway. 


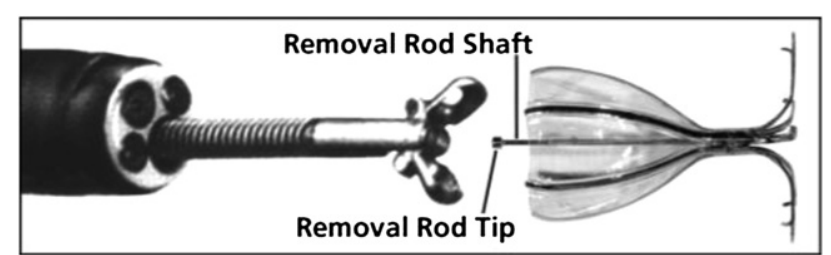

FIGURE 5. Diagram showing intrabronchial valve removal. Provided by Spiration Inc (Redmond, Wash).

group compared with the control groups $(P=.02)$. Finally, exacerbations of chronic obstructive pulmonary disease requiring hospitalization occurred more often in the EBV group $(7.9 \%)$ than in the control group $(1.1 \%, P=.03)$ but at a similar rate during late follow-up $(P=.84)$.

Our institutional experience involved approximately 25 patients with placement of IBVs for PALs. The etiologies for our cohort ranged from spontaneous pneumothoraces to inadvertent placement of chest tubes into the lung parenchyma. In most cases, because the IBVs were ultimately removed once the air leak had resolved (typically 6 weeks after placement), many of the complications described in the VENT trial were infrequently seen in our experience using IBVs for PALs. No deaths, cardiovascular complications, pulmonary complications, or implant-related events have been related to the placement of IBVs. Although rare, the most common unexpected event after valve placement was failure to completely resolve the PAL. In the few cases without complete resolution, the degree of the air leak was significantly reduced. The postulated explanation for the inability to completely resolve the identified leaks was the presence of collateral ventilation. In such instances, the patients should undergo placement of a pigtail catheter into the pleural space, with attachment to a dry seal chest drain and discharge home with appropriate follow-up.

\section{CONCLUSIONS}

The off-label use of airway valves for the treatment of PALs has emerged as an increasingly popular therapeutic

\section{TABLE 1. Possible complications of IBV placement}

\section{Atelectasis}

Bleeding observed from an airway treated with an IBV

Bronchitis

Damage in the airway and/or tissue near an IBV

Infection distal to IBV

Local airway swelling or edema at site of IBV implantation

Migration of IBV out of airway or lung

Persistent cough

Pneumothorax

Shortness of breath

Tissue hyperplasia or granulation at site of IBV implantation Death

$I B V$, Intrabronchial valve. option. ${ }^{5,7,8,17,18}$ The use of IBVs continues to be a safe and effective option in the hands of experienced operators. The placement of IBVs is a multistep process requiring a detailed understanding of the airway anatomy and a systematic approach to air leak isolation. Although no formal data have been published regarding the cost/ benefit analysis of IBV use in patients with PALs secondary to APFs, the reduction of hospital days observed in patients who have received IBVs compared with those receiving conventional therapy is likely to demonstrate the cost effectiveness. The use of IBVs in the treatment of PALs by interventional pulmonologists allows for increased awareness of the devices' capabilities in other specialties, such as thoracic surgery. IBVs represent minimally invasive technology capable of providing thoracic surgeons with an effective therapeutic option for the treatment of PALs.

\section{References}

1. Wood DE, McKenna RJ, Yusen RD. A multicenter trial of an intrabronchial valve for treatment of severe emphysema. J Thorac Cardiovasc Surg. 2007; 133:65-73.

2. Sterman D, Mehta A, Wood D. A multicenter pilot study of a bronchial valve for the treatment of severe emphysema. Respiration. 2010;79:222-33.

3. Toma T, Hopkinson N, Hillier J. Unilateral bronchoscopic volume reduction with valve implants in patients with severe emphysema. Lancet. 2003;361: 931-3.

4. Sciurba FC, Ernst A, Herth F. A randomized study of endobronchial valves for advanced emphysema. N Engl J Med. 2010;363:1233-44.

5. Gillespie CT, Sterman D, Cerfolio R. Endobronchial valve treatment for prolonged air leaks of the lung: a case series. Ann Thorac Surg. 2011;91: 270-3.

6. Travaline J, McKenna RJ, De Giacoma T, Venuta F, Hazelrigg SR, Boomer M, et al. Treatment of persistent pulmonary air leaks using endobronchial valves. Chest. 2009;136:355-60.

7. Mahajan A, Verhoef P, Patel SB, Carr G, Hogarth DK. Intrabronchial valves: a case series describing a minimally invasive approach to bronchopleural fistulas in medical intensive care unit patients. J Bronchol Intervent Pulmonol. 2012;19:137-41.

8. El-Sameed Y, Waness A, Al Shamsi I, Mehta AC. Endobronchial valves in the management of broncho-pleural and alveolo-pleural fistulae. Lung. 2012;190: 347-51.

9. Williams N, Lewis C. Bronchopleural fistula: a review of 86 cases. Br J Surg. 1976;63:520-2.

10. Malave G, Foster E, Wilson J. Bronchopleural fistula-present-day study of an old problem: a review of 52 cases. Ann Thorac Surg. 1971;11:1-10.

11. Chee C, Abisheganadan J, Yeo J. Persistent air leak in spontaneous pneumothorax-clinical course and outcome. Respir Med. 1998;92:757-61.

12. Kempainen R, Pierson D. Persistent air leaks in patients receiving mechanical ventilation. Semin Respir Crit Care Med. 2001;22:675-84.

13. Tobin M. Principle and practice of mechanical ventilation. 2nd ed. New York: McGraw-Hill; 2006.

14. Fruchter O, Bruckheimer E, Raviv Y, Rosengarten D, Saute M, Kramer M. Endobronchial closure of bronchopleural fistulas with Amplatzer vascular plug. Eur J Cardiothorac Surg. 2011;41:46-9.

15. Hull M, Nader D, Fullingim J. The diagnosis and localization of a bronchopleural fistula using single proton emission computer tomography (SPECT) imaging. Chest. 2008;134:c18001-c18001.

16. York E, Lewall D, Hirji M, Gelfand E, Modry D. Endoscopic diagnosis and treatment of postoperative bronchopleural fistula. Chest. 1990;97:1390-2.

17. Schiavon M, Marulli G, Zuin A, Nicotra S, Di Chiara F, De Filippis F, et al. Endobronchial valve for secondary pneumothorax in a severe emphysema patient. Thorac Cardiovasc Surg. 2011;59:509-10.

18. Wood D, Cerfolio R, Gonzalez X, Springmeyer S. Bronchoscopic management of prolonged air leak. Clin Chest Med. 2010;31:127-33. 